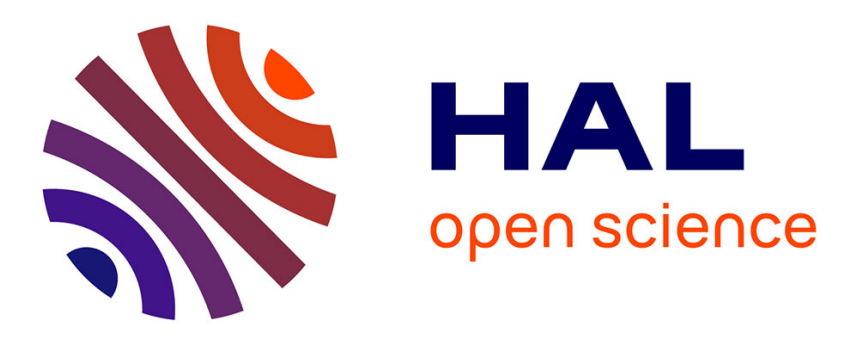

\title{
Extremal Graphs with respect to the Modified First Zagreb Connection Index
}

\author{
Guillaume Ducoffe, Ruxandra Marinescu-Ghemeci, Camelia Obreja, \\ Alexandru Popa, Rozica Maria Tache
}

\section{- To cite this version:}

Guillaume Ducoffe, Ruxandra Marinescu-Ghemeci, Camelia Obreja, Alexandru Popa, Rozica Maria Tache. Extremal Graphs with respect to the Modified First Zagreb Connection Index. 20th International Symposium on Symbolic and Numeric Algorithms for Scientific Computing (SYNASC), Sep 2018, Timisoara, Romania. 10.1109/SYNASC.2018.00033 . hal-02011274

\section{HAL Id: hal-02011274 https://hal.science/hal-02011274}

Submitted on 7 Feb 2019

HAL is a multi-disciplinary open access archive for the deposit and dissemination of scientific research documents, whether they are published or not. The documents may come from teaching and research institutions in France or abroad, or from public or private research centers.
L'archive ouverte pluridisciplinaire HAL, est destinée au dépôt et à la diffusion de documents scientifiques de niveau recherche, publiés ou non, émanant des établissements d'enseignement et de recherche français ou étrangers, des laboratoires publics ou privés. 


\section{Extremal Graphs with respect to the Modified First Zagreb Connection Index}

\author{
Guillaume Ducoffe \\ National Institute for Research and \\ Development in Informatics \\ The Research Institute of the University of Bucharest \\ Bucharest, Romania \\ Email: guillaume.ducoffe@ici.ro \\ Alexandru Popa \\ University of Bucharest \\ National Institute for Research and Development in Informatics \\ Bucharest, Romania \\ Email: alexandru.popa@fmi.unibuc.ro
}

\author{
Ruxandra Marinescu-Ghemeci \\ University of Bucharest \\ Bucharest, Romania
}

Email:verman@fmi.unibuc.ro

\author{
Camelia Obreja \\ University of Bucharest \\ Bucharest, Romania
}

Email: camelia.obreja@gmail.com

\author{
Rozica Maria Tache \\ University of Bucharest, Romania \\ Bucharest, Romania \\ Email: rozica.tache@fmi.unibuc.ro
}

\begin{abstract}
Topological indices (TIs) have an important role in studying properties of molecules. A main problem in mathematical chemistry is finding extreme graphs with respect to a given TI. In this paper extremal graphs with respect to the modified first Zagreb connection index for trees in general and for trees with given number of pendants, for unicyclic graphs with or without a fixed girth and connected graphs are determined, using methods with higher degree of generality with respect to the transformation techniques usually used in such context. These graphs are relevant for chemical studies.
\end{abstract}

Keywords-topological index; Zagreb index; trees; pendants; unicyclic graphs

\section{INTRODUCTION}

Molecular descriptors are numerical values that characterize different properties of molecules. They are used mainly in the construction of Quantitative Structure-Activity Relationship (QSAR) or Quantitative Structure-Property Relationship (QSPR) models to generate predictions about the biological activity or physico-chemical properties of new untested or even yet to be synthetized substances. Such models have an accuracy up to $90 \%$ in predicting the effects of different medicines on some species of parasites which cause a large number of deaths or viruses known for their aggressivity, like Hepatitis B and C, Cytomegalovirus and HIV-1 [4], [5]. Moreover, these models provides new drugs much more active than those already existing on the market [2]. A special family of molecular descriptors is represented by the topological indices (TIs), invariants that characterize the topology of a graph, indicating its ramification. TIs are mainly studied in connection with chemical graphs, which are undirected, connected graphs that model organic compounds by considering only the carbon-carbon and carbonhydrogen type atomic bonds. Analysed in this context, the TIs appeared from the desire of studying structural properties of chemical compounds. Since the structures of substances, even modelled as graphs, cannot be readily investigated as such, numerical quantities associated with these structures have to be considered for successful studies in this area [2], [4], [5]. Thus TIs participate, along with other molecular descriptors, in the construction of QSAR or QSPR models that generate predictions about the biological activity or physico-chemical properties of new substances. Consequently, more and more topological indices are being introduced in the literature with the purpose of being used mainly in applications within different areas of chemistry.

One of the most important and intensely studied TIs are the Zagreb indices, introduced in [6] from the desire to examine the dependence of the total $\pi$-electron energy on the molecular structure. They are defined by $M_{1}(G)=$ $\sum_{v \in V(G)} d_{G}(v)^{2}$ and $M_{2}(G)=\sum_{(u, v) \in E(G)} d_{G}(u) d_{G}(v)$, where $G=(V(G), E(G))$ is a connected graph and $d_{G}(v)$ denotes the degree of the vertex $v$.

Starting from these, a whole family of Zagreb indices appeared, including the multiplicative Zagreb indices [8], the reformulated Zagreb indices [9] and the modified Zagreb connection index [6]. This class of indices has been used to study molecular complexity, chirality, $Z E$-isomerism, heterosystems and has been used with good results in QSAR and QSPR models. Thus, mathematical chemistry experts note that the best descriptor models considered in the literature contains the Zagreb $M_{2}$-index [7].

An important direction in the field of mathematical chemistry is given by the determination of the extreme graphs with respect to a given TI. This field started with the leading article of Bollobás and Erdös [3], in which they determine the extremal graphs with respect to the Randić index, a wellknown and intensely used in QSAR and QSPR models TI.

The main method used in determining the extremal graphs is defining graph transformations that, under certain condi- 
tions, strictly increase/decrease the value of the topological index. This leads us to the extremal graph(s) by the following principle: whenever a graph is not yet of the desired shape, there is a way to apply one of the transformations mentioned above to yield a new graph; since the class of graphs under investigation is finite, this process is finite; since the process gives the same end result regardless of the graph it starts with, it exhibits the extremal graph(s) of the class in question.

\section{OUR RESUlTS}

In this paper we study a modified Zagreb index introduced by Gutman and Trinajstić in [6], but studied for the first time in [1], and named the modified first Zagreb connection index (also called $Z C_{1}^{*}$-index):

$$
Z C_{1}^{*}(G)=\sum_{v \in V(G)} d_{G}(v) \tau_{G}(v)
$$

where $\tau_{G}(v)$ denotes the number of vertices at distance 2 from $v$, also called the connection number of $v$. The latter article determines the extremal graphs with respect to the $Z C_{1}^{*}$-index in the class of chemical trees (in which every vertex has degree at most four) and the tree having minimum $Z C_{1}^{*}$-index. These are the only known results on this index, the trees that maximize the $Z C_{1}^{*}$-index were not determined nor other classes of graphs have been studied until our work.

In this paper we extend the above study by considering general trees, trees with $k$ pendants and unicyclic graphs with given or arbitrary girth, all of them representing classes of graphs of interest for the field of mathematical chemistry, due to their topology that closely resembles molecular structures.

We propose a new method with higher degree of generality with respect to the transformation techniques usually used in such context. It allows to find transformations that strictly increases or decreases the index by seeing the graph as a result of union operations of subgraphs and study the changes in the index when replacing a subgraph with another. This is useful, since various types of decomposition of graphs exists in literature, such as block decompositions.

The paper is structured as follows. In Section III we introduce the vertex-union operation and present some results on $Z C_{1}^{*}$-index for this operation, used in the next sections in order to provide extremal graphs with respect to $Z C_{1}^{*}$ index. In Section IV we study maximal trees, completing the results from [1]. Moreover, maximal graphs with respect to $Z C_{1}^{*}$ in the class of trees with fixed number of pendants are determined. Next section is dedicated to unicyclic graphs, first with fixed girth and then with arbitrary girth. We determine extremal graphs - both minimal and maximal with respect to $Z C_{1}^{*}$ for these families of graphs. In Section VI the general case of connected graphs is considered. We prove necessary and sufficient conditions for a connected graph to maximize the $Z C_{1}^{*}$-index.

\section{III. $Z C_{1}^{*}$-INDEX FOR GRAPH OPERATIONS AND TRANSFORMATIONS}

In this section we consider a graph operation that we call vertex-union and study the $Z C_{1}^{*}$-index for graphs obtained using that operation, in order to be able to easier find transformations on graphs that strictly increase or decrease the $Z C_{1}^{*}$-index.

Let $G, H$ be two connected graphs and consider one vertex from each graph: $a \in V(G), b \in V(H)$. Denote by $(G, a) \odot(H, b)$ the graph obtained from the union of graphs $G$ and $H$ by identifying vertices $a$ and $b$. We will call this operation vertex-union.

Note that if a graph has cut vertices, it can be seen as a vertex-union of its biconnected components.

For a graph $G$ denote by $d(G)$ the set of the degrees of its vertices. For a vertex $v \in V(G)$ denote by $s_{G}(v)$ the sum of the degrees of its neighbors, by $\operatorname{ecc}_{G}(v):=$ $\max \{d(v, u) \mid u \in V\}$ the eccentricity of vertex $v$ and by $s t_{G}(v)=\tau_{G}(v)+s_{G}(v)$. A vertex is call pendant if it has degree 1. A pendant edge is an edge incident in a pendant vertex.

Remark 1: If $G$ is a tree and $v$ is a vertex in $G$, then

$$
s_{G}(v)=d_{G}(v)+\tau_{G}(v) .
$$

Let $B$ be an induced subgraph of $G$. We call $B$ a pendant subgraph of $G$ with root $y$ if $G=(G-(B-y), y) \odot(B, y)$ (there are no edges with one extremity in $V(B)-\{y\}$ and other in $V(G-B), y$ being a cut vertex).

Note that an end block of a graph is a pendant subgraph.

A pendant path $P$ in $G$ is then a pendant subgraph of $G$ with root $y$ isomorphic to a path, such that $d_{G}(y) \geq 3$ and $y$ is an extremity of $P$. Thus, each internal vertex of a pendant path has degree 2 in $G$ and one extremity is a pendant vertex in $G$ (the other extremity being $y$ ).

For two vertices $y$ and $u$, denote:

$$
\gamma_{G, y, u}= \begin{cases}0, & \text { if } u \text { and } y \text { are adjacent }, \\ 1, & \text { otherwise. }\end{cases}
$$

In order to determine the $Z C_{1}^{*}$-index for graphs obtained from vertex-union operations, we define the following constants:

$$
\epsilon_{n}=\left\{\begin{array}{ll}
4, & \text { if } n \geq 5, \\
2, & \text { if } n=4, \\
0, & \text { if } n=3
\end{array} \quad \alpha_{n}= \begin{cases}6, & \text { if } n \geq 5 \\
5, & \text { if } n=4 \\
4, & \text { if } n=3\end{cases}\right.
$$

Lemma 1: Let $G, H$ be two connected graphs and two vertices $a \in V(G), b \in V(H)$. Let $\Omega=(G, a) \odot(H, b)$. Then

$$
\begin{aligned}
Z C_{1}^{*}(\Omega)= & Z C_{1}^{*}(G)+Z C_{1}^{*}(H)+\left(s_{G}(a)+\tau_{G}(a)\right) d_{H}(b)+ \\
& +d_{G}(a)\left(s_{H}(b)+\tau_{H}(b)\right) .
\end{aligned}
$$


Proof: By definition, the formula for $Z C_{1}^{*}(\Omega)$ is:

$$
\begin{aligned}
Z C_{1}^{*}(\Omega)= & \sum_{x \in V(\Omega)} d_{\Omega}(x) \tau_{\Omega}(x)= \\
= & \sum_{x \in V(G)-\{a\}} d_{\Omega}(x) \tau_{\Omega}(x)+d_{\Omega}(a) \tau_{\Omega}(a)+ \\
& +\sum_{y \in V(H)-\{b\}} d_{\Omega}(y) \tau_{\Omega}(y)
\end{aligned}
$$

For a vertex $x \in V(G)$ we have:

- $d_{\Omega}(x)=d_{G}(x), \tau_{\Omega}(x)=\tau_{G}(x)$, if $x \notin\{a\} \cup N_{G}(a)$

- $d_{\Omega}(x)=d_{G}(x), \tau_{\Omega}(x)=\tau_{G}(x)+d_{H}(b)$, if $x \in N_{G}(a)$

- $d_{\Omega}(x)=d_{G}(x)+d_{H}(b), \tau_{\Omega}(x)=\tau_{G}(x)+\tau_{H}(b)$, if $x=a$

For a vertex $y \in V(H)$ we have:

- $d_{\Omega}(y)=d_{H}(y), \tau_{\Omega}(y)=\tau_{H}(y)$, if $y \notin\{b\} \cup N_{H}(b)$

- $d_{\Omega}(y)=d_{H}(y), \tau_{\Omega}(y)=\tau_{H}(y)+d_{G}(a)$, if $y \in N_{H}(b)$

By replacing these relations in $Z C_{1}^{*}(\Omega)$ formula, the stated result is obtained.

Since for the classes of graphs considered in this paper are results of applying vertex-union operations on trees and cycles or of moving pendant edges from one vertex to another, we derive formulas for the case when one of the graphs is a tree, a path or a cycle.

Corollary 1: Let $G, H$ be two connected graphs and two vertices $a \in V(G), b \in V(H)$. Let $\Omega=(G, a) \odot(H, b)$. If $H$ is a tree, then

$$
\begin{aligned}
Z C_{1}^{*}(\Omega)= & Z C_{1}^{*}(G)+Z C_{1}^{*}(H)+2 s_{H}(b) d_{G}(a)+ \\
& +d_{H}(b)\left(s_{G}(a)+\tau_{G}(a)-d_{G}(a)\right) .
\end{aligned}
$$

Proof: By Remark 1 we have $s_{H}(b)+\tau_{H}(b)=$ $2 s_{H}(b)-d_{H}(b)$. Using this relation and Lemma 1 the result follows.

Corollary 2: Let $G, H$ be two connected graphs and two vertices $a \in V(G), b \in V(H)$. Let $\Omega=(G, a) \odot(H, b)$.

- If $H \simeq P_{2}$, then

$$
Z C_{1}^{*}(\Omega)=Z C_{1}^{*}(G)+d_{G}(a)+\tau_{G}(a)+s_{G}(a) .
$$

- If $H \simeq P_{n}, n \geq 3$, then

$$
Z C_{1}^{*}(\Omega)=Z C_{1}^{*}(G)+Z C_{1}^{*}\left(P_{n}\right)+3 d_{G}(a)+\tau_{G}(a)+s_{G}(a) .
$$

- If $H \simeq C_{n}, n \geq 3$, then

$Z C_{1}^{*}(\Omega)=Z C_{1}^{*}(G)+\epsilon_{n} n+\alpha_{n} d_{G}(a)+2 \tau_{G}(a)+2 s_{G}(a)$

Proof: We apply Lemma 1 . If $H \simeq P_{2}$, then $Z C_{1}^{*}(H)=0, d_{H}(b)=s_{H}(b)=1$ and $\tau_{H}(b)=0$.

If $H \simeq P_{n}, n \geq 3$ then $d_{H}(b)=1, s_{H}(b)=2$ and $\tau_{H}(b)=1$. If $H \simeq C_{n}$ then $Z C_{1}^{*}(H)=n d_{H}(b) \tau_{H}(b)=$ $\epsilon_{n} n, d_{H}(b)=2, s_{H}(b)=4$ and

$$
\tau_{H}(b)= \begin{cases}2, & \text { if } n \geq 5 \\ 1, & \text { if } n=4 \\ 0, & \text { if } n=3\end{cases}
$$

Let $G$ be a graph, $B$ a pendant subgraph of $G$ rooted at $y$ and $u \in V(G)-V(B)$. Denote $N_{B}(y)=\left\{y_{1}, \ldots, y_{p}\right\}$. We define the following transformation $t$ on $G$ through which we obtain the graph:

$$
t(G, B, y, u)=G-\left\{y y_{1}, \ldots, y y_{p}\right\} \cup\left\{u y_{1}, \ldots, u y_{p}\right\}
$$

by removing the pendant subgraph $B$ from $y$ and attaching it to vertex $u$.

Remark 2: With the notations used for defining transformation $t(G, B, y, u)$, let $G_{e}=G-(B-y)$.

We have $G=\left(G_{e}, y\right) \odot(B, y)$ and $t(G, B, y, u)=$ $\left(G_{e}, u\right) \odot(B, y)$

Lemma 2: Let $G$ be a connected graph and $B$ a pendant subgraph of $G$ rooted at $y$. Then

$$
\begin{aligned}
& Z C_{1}^{*}(t(G, B, y, u))-Z C_{1}^{*}(G)= \\
& =d_{B}(y)\left(s t_{G}(u)-s t_{G}(y)\right)+s t_{B}(y)\left(d_{G}(u)-d_{G}(y)\right)+ \\
& \quad+2 d_{B}(y)\left(s t_{B}(y)-\left(1-\gamma_{G, y, u}\right) d_{B}(y)\right)
\end{aligned}
$$

Proof: Let $G_{e}=G-(B-y)$. By Remark 2 and Lemma 1 we obtain:

$$
\begin{aligned}
& Z C_{1}^{*}(t(G, B, y, u))-Z C_{1}^{*}(G)= \\
& =Z C_{1}^{*}\left(\left(G_{e}, u\right) \odot(B, y)\right)-Z C_{1}^{*}\left(\left(G_{e}, y\right) \odot(B, y)\right)= \\
& =\left(d_{G_{e}}(u)-d_{G_{e}}(y)\right)\left(s_{B}(y)+\tau_{B}(y)\right)+ \\
& \quad+\left(\tau_{G_{e}}(u)-\tau_{G_{e}}(y)\right) d_{B}(y)+\left(s_{G_{e}}(u)-s_{G_{e}}(y)\right) d_{B}(y) .
\end{aligned}
$$

We have

- $d_{G_{e}}(u)=d_{G}(u)$ and $d_{G_{e}}(y)=d_{G}(y)-d_{B}(y)$

- $s_{G_{e}}(u)=s_{G}(u)-\left(1-\gamma_{G, y, u}\right) d_{B}(y)$ and $s_{G_{e}}(y)=$ $s_{G}(y)-s_{B}(y)$

- $\tau_{G_{e}}(u)=\tau_{G}(u)-\left(1-\gamma_{G, y, u}\right) d_{B}(y)$ and $\tau_{G_{e}}(y)=$ $\tau_{G}(y)-\tau_{B}(y)$.

By replacing this relations into the above formula for $Z C_{1}^{*}(t(G, B, y, u))-Z C_{1}^{*}(G)$, the result follows.

Corollary 3: Let $G$ be a connected graph and $B$ a pendant subgraph of $G$ rooted at $y$.

1) If $B \simeq P_{2}$ then $Z C_{1}^{*}\left(t\left(G, P_{2}, y, u\right)\right)-Z C_{1}^{*}(G)=$ $\left(s_{G}(u)-s_{G}(y)\right)+\left(\tau_{G}(u)-\tau_{G}(y)\right)+\left(d_{G}(u)-d_{G}(y)\right)+$ $2 \gamma_{G, y, u}$.

2) If $B \simeq C_{p}$ then $Z C_{1}^{*}\left(t\left(G, C_{p}, y, u\right)\right)-Z C_{1}^{*}(G)=$ $2\left(s_{G}(u)-s_{G}(y)\right)+2\left(\tau_{G}(u)-\tau_{G}(y)\right)+\alpha_{p}\left(d_{G}(u)-\right.$ $\left.d_{G}(y)\right)+4 \alpha_{p}-8\left(1-\gamma_{G, y, u}\right)$.

3) If $B \simeq P_{q}$ with $q>2$ then $Z C_{1}^{*}\left(t\left(G, P_{q}, y, u\right)\right)-$ $Z C_{1}^{*}(G)=\left(s_{G}(u)-s_{G}(y)\right)+\left(\tau_{G}(u)-\tau_{G}(y)\right)+$ $3\left(d_{G}(u)-d_{G}(y)\right)+4+2 \gamma_{G, y, u}$.

Proof: The result follows from Lemma 2 by replacing the values for $d_{B}(y), \tau_{B}(y)$ and $s_{B}(y)$. Note that $\tau_{C_{p}}(y)=$ $\alpha_{p}-4$.

\section{TREES}

\section{A. General trees}

In [1] it is proven that, for $n \geq 5$, the path graph $P_{n}$ has minimum $Z C_{1}^{*}$ value among all trees with $n$ vertices and this minimum value is $4 n-10$. In this section the trees with $n$ vertices having maximum $Z C_{1}^{*}$ value are determined. Let $\mathcal{S}_{n}$ be the family of trees with $n$ vertices and diameter at most 3 . Note that the only trees in $\mathcal{S}_{n}$ are the star $S_{n-1}$ and 
double stars $S_{p, q}$ with $p, q>0, p+q=n-2$, obtained from the union of stars $S_{p}$ and $S_{q}$ by adding an edge joining their centers.

Lemma 3: Let $n \geq 4$ and $p, q$ two positive integers with $p+q=n-2$. Then

$$
Z C_{1}^{*}\left(S_{n-1}\right)=Z C_{1}^{*}\left(S_{p, q}\right)=(n-2)(n-1) .
$$

Proof: The result follows from the definition of $Z C_{1}^{*}$.

Theorem 1: The trees with $n \geq 4$ vertices having the maximum value of modified first Zagreb connection index are the trees in $\mathcal{S}_{n}$.

Proof: Let $T \notin \mathcal{S}_{n}$ be an arbitrary tree with $n$ vertices. We prove that there is a tree having a greater $Z C_{1}^{*}$ value than $T$, obtained by applying a transformation of type $t$ to $T$ as follows. Let $x, x^{\prime}$ be two vertices at distance equal to $\operatorname{diam}(T)$. Note that $\operatorname{diam}(T) \geq 4$. Then $x, x^{\prime}$ are pendant vertices and $d\left(x, x^{\prime}\right) \geq 4$. Let $y, u$ the only neighbors of vertices $x$ and $x^{\prime}$, respectively. Then $d(u, y) \geq 2$. Assume wlog that $s_{T}(u) \geq s_{T}(y)$. Consider the tree $T^{\prime}=t(T, P, y, u)$ where $P=[y, x]$. By Corollary 3 and Remark 1 we have

$$
\begin{aligned}
& Z C_{1}^{*}\left(T^{\prime}\right)-Z C_{1}^{*}(T)= \\
& =\left(s_{T}(u)+\tau_{T}(u)+d_{T}(u)\right)- \\
& \left.\quad-\left(s_{T}(y)\right)+\tau_{T}(y)+d_{T}(y)\right)+2= \\
& =2\left(s_{T}(u)-s_{T}(y)\right)+2>0,
\end{aligned}
$$

hence $T$ is not a maximal tree with respect to $Z C_{1}^{*}$.

It follows that the only trees than can maximize $Z C_{1}^{*}$ are in the family $\mathcal{S}_{n}$. By Lemma 3 all the trees in $\mathcal{S}_{n}$ have the same first modified Zagreb connection index, hence they maximize $Z C_{1}^{*}$.

Theorem 2: [1] The tree with $n \geq 4$ vertices having the minimum value of modified first Zagreb connection index is the path $P_{n}$, with $Z C_{1}^{*}\left(P_{n}\right)=4 n-10$.

\section{B. Trees with $k$ pendants}

One relevant class of trees for chemical studies are the trees with given number of pendants. In this section we determine the trees with $k$ pendants that maximize the $Z C_{1}^{*}$ index. Note that if $k=2$ there is only one tree with $n$ vertices and $k$ pendants, namely the path. Assume next that $k \geq 3$.

A spider tree is a tree in which all pendant paths are rooted in the same vertex $u$, that is all vertices except $u$ have degree at most 2 .

Denote by $\mathcal{T}_{n, k, \leq 2}$ the set of spider trees with $n$ vertices and $k$ pendants such that all pendant paths have length at most 2 and by $\mathcal{T}_{n, k, \geq 2}$ the set of spider trees with $n$ vertices such that all pendant paths have length at least 2 .

Denote by $\mathcal{S}_{n}^{d}=\mathcal{S}_{n}-S_{n-1}$ the family of double stars with $n$ vertices.

Lemma 4: a) There is only one spider tree in $\mathcal{T}_{n, k, \leq 2}$ and it has the $Z C_{1}^{*}$-index equal to $2 n k-k^{2}-3 k$.

b) Let $T$ be a spider tree in $\mathcal{T}_{n, k, \geq 2}$. Then $Z C_{1}^{*}(T)=$ $4 n+3 k^{2}-9 k-4$.
Proof: a) A tree in $\mathcal{T}_{n, k, \leq 2}$ with $r$ pendant paths of length 2 has $2 r+(k-r)+1=k+r+1$ vertices, hence $r=n-k-1$. Thus, the only tree $T$ in $\mathcal{T}_{n, k, \leq 2}$ is the spider with $r=n-k-1$ pendant paths of length 2 and $k-r$ paths of length 1 . Then, by definition of the $Z C_{1}^{*}$-index, we have $Z C_{1}^{*}(T)=k \cdot r+r(2(k-1))+r+(k-r)(k-1)=$ $2 n k-k^{2}-3 k$.

b) Let $T \in \mathcal{T}_{n, k, \geq 2}$. Let $P_{l}, l \geq 4$ be a pendant path with $l$ vertices in $T$ with root $u$ and let $x$ be an extremity of degree 1 of another pendant path of $T$. Denote by $y$ the pendant extremity of $P_{l}$ and by $z$ the neighbor of $y$ in $P_{l}$. Then $s_{T}(z)=3$ and $s_{T}(x)=2$. The tree $T^{\prime}=t(T, P, z, x)$ with $P=[y, z]$ obtained by moving the pendant edge from $P_{l}$ in pendant vertex $x$ has also $k$ pendant vertices and, by Corollary 3 and Remark $1, Z C_{1}^{*}\left(T^{\prime}\right)-Z C_{1}^{*}(T)=2\left(s_{T}(x)-\right.$ $\left.s_{T}(z)\right)+2=0$, hence $Z C_{1}^{*}(t(T, P, z, x))=Z C_{1}^{*}(T)$.

It can be easily seen that if $T_{1}$ and $T_{2}$ are two trees in $\mathcal{T}_{n, k, \geq 2}, T_{2}$ can be obtained from $T_{1}$ by successively moving a pendant edge from a pendant path of length at least 3 to a pendant extremity of another pendant path of length at least 2, thus $Z C_{1}^{*}\left(T_{1}\right)=Z C_{1}^{*}\left(T_{2}\right)$.

Denote by $Z(n, k)$ the $Z C_{1}^{*}$-index of a tree from $\mathcal{T}_{n, k, \geq 2}$. Then, by Lemma 1 and from the fact that there is at least one tree in $\mathcal{T}_{n, k, \geq 2}$ having a pendant path of length 2 we obtain the following recursive formula: $Z(n, k)=Z(n-2, k-1)+$ $Z C_{1}^{*}\left(P_{3}\right)+1 \cdot(3(k-1))+3(k-1)=Z(n-2, k-1)+6 k-4$. Since $Z(n, 2)=Z C_{1}^{*}\left(P_{n}\right)=4 n-10$ (Theorem 2) we obtain

$$
Z(n, k)=Z(n-2(k-2), 2)-4(k-2)+6 \sum_{i=3}^{k} i
$$

and thus $Z(n, k)=4 n+3 k^{2}-9 k-4$.

Theorem 3: The trees with $n \geq 4$ vertices and $k>2$ pendants having the maximum value of modified first Zagreb connection index are the trees in $\mathcal{T}_{n, k, \leq 2} \cup \mathcal{S}_{n}^{d}$ if $k=n-2$, in $\mathcal{T}_{n, k, \leq 2}$, if $n-1 \leq 2 k, k \neq n-2$ and in $\mathcal{T}_{n, k, \geq 2}$ otherwise.

Proof: Let $T$ be a tree with $n \geq 4$ vertices and $k$ pendants such that it maximizes $Z C_{1}^{*}$-index. We have the following situations.

Case 1. There are two distinct vertices $y, u$ in which are incident pendant paths of length at least 2. Assume wlog that $3 d_{T}(u)+s_{T}(u)+\tau_{T}(u) \geq 3 d_{T}(y)+s_{T}(y)+\tau_{T}(y)$ and let $P_{l}$ be a pendant path of length at least 2 incident in $y$. Consider $T^{\prime}=t\left(T, P_{l}, y, u\right)$. Then $T^{\prime}$ is a tree with $k$ pendants. By Corollary 3 we obtain that $Z C_{1}^{*}\left(T^{\prime}\right)-Z C_{1}^{*}(T) \geq$ $4+2 \gamma_{G, y, u}>0$, contradiction.

It follows that all pendant paths of length at least 2 , if exist, are incident in the same vertex. Denote by $u$ this vertex.

Case 2. There are two distinct vertices $x, y$ with $d(x, y) \geq 2$ in which are incident pendant paths of length 1 (which are pendant edges). By Corollary 3, as in proof of Theorem 1, by applying transformation $t$ we can obtain a tree $t\left(T, P_{2}, y, u\right)$ with $k$ pendants having a greater $Z C_{1}^{*}$-index, contradiction. 
It follows that all pendant paths of length 1 are incident in one vertex or in two adjacent vertices.

Case 3. Assume there is at least one pendant path $P_{l}$ of length $l \geq 2$ rooted in $u$ and only one vertex $x$ different from $u$ in which are incident pedant paths of length 1 . Denote by $p$ the number of pendant paths of length 1 incident in $x$. Note that $p>1$ and all vertices different from $x$ and $u$ have degree at most two, since all pendant paths are rooted in $x$ or $u$. Also, since $u$ has degree greater than 2 , there are $q>1$ pendant paths rooted at $u$.

Subcase 3.1. Assume first that $u$ and $x$ are not adjacent.

Then the vertices from $N_{G}(x)$ are pendant with one exception, a vertex $z$ of degree 2 .

Let $P$ be a pendant path rooted in $u$ different from $P_{l}$ and let $B$ be the pendant subgraph induced by all the pendant paths different from $P$ that are rooted in $u$. Let $T_{B}=T-$ $(V(B)-\{u\})$. We have $T=\left(T_{B}, u\right) \odot(B, u)$. Consider $T^{\prime}=\left(T_{B}, x\right) \odot(B, u)=t(T, B, u, x)$. Then by Lemma 1 we obtain:

$$
\begin{aligned}
Z C_{1}^{*}\left(T^{\prime}\right)-Z C_{1}^{*}(T)= & d_{B}(u)\left(s t_{T_{B}}(x)-s t_{T_{B}}(u)\right)+ \\
& +s t_{B}(u)\left(d_{T_{B}}(x)-d_{T_{B}}(u)\right)
\end{aligned}
$$

But $d_{T_{B}}(x)=p+1>d_{T_{B}}(u)=2, s t_{T_{B}}(x)=1+$ $(p+2)=p+3$ and $s t_{T_{B}}(u) \leq 2+4=6$. If $p>2$, then $s t_{T_{B}}(x) \geq s t_{T_{B}}(u)$ and we have $Z C_{1}^{*}\left(T^{\prime}\right)>Z C_{1}^{*}(T)$. Otherwise $p=2$ and then

$$
Z C_{1}^{*}\left(T^{\prime}\right)-Z C_{1}^{*}(T) \geq s t_{B}(u)-d_{B}(u) .
$$

But, since $P_{l}$ is a pendant path in $B$ rooted in $u$ of length at least 2 , vertex $u$ has a neighbor of degree at least 2 in $B$, hence $s t_{B}(u)-d_{B}(u)>0$.

It follows that in this case $T$ does not maximize the $Z C_{1}^{*}$ index.

Subcase 3.2. Assume now that $u$ and $x$ are adjacent. Then, since in $u$ is incident at least one pendant path of length at least 2 , we have $s_{T}(u)>p+1+q$ and $s_{T}(x)=p+1+q$. Then, from Corollary 3 and Remark 1, it follows that by moving a pendant edge $x y$ from $x$ to $u$ we obtain a tree with $k$ pendants having a greater index than $T$, more exactly: $Z C_{1}^{*}(t(T,[x, y], x, u))-Z C_{1}^{*}(T)=2\left(s_{T}(u)-s_{T}(x)\right)>0$, hence the $Z C_{1}^{*}$-index is not maximized in this subcase either. Case 4. There are two adjacent vertices $x$ and $y$ in which are incident $p$, respectively $q$ pendant paths of length 1 and no other pendant paths are incident in $x$ or $y$. Then all vertices from $N_{G}(x)-\{y\}$ and $N_{G}(y)-\{x\}$ have degree one with at most one exception.

If all have degree one, then $T$ is a double star and, by Theorem 1, $T$ maximizes the $Z C_{1}^{*}$-index.

Otherwise, assume there is $z \in N_{G}(y)$ of degree at least 2. Then $p=\left|N_{G}(x)-\{y\}\right|>1$ and the vertices from $N_{G}(x) \cup N_{G}(y)$ induce a pendant subgraph $H \simeq S_{p, q+1}$ rooted at $z$ and we have $T=\left(S_{p, q+1}, z\right) \odot\left(T_{H}, z\right)$ where $T_{H}=T-(V(H)-\{z\})$.

Consider $T^{\prime}=\left(S_{1, p+q}, a\right) \odot\left(T_{H}, z\right)$, where $a$ is a pendant vertex in $S_{1, p+q}$ adjacent with the center of degree $p+q+2$.
$T^{\prime}$ has also $k$ pendant vertices. By Lemma 3 we have $Z C_{1}^{*}\left(S_{1, p+q}\right)=Z C_{1}^{*}\left(S_{p, q+1}\right)$. Then, from Lemma 1 it follows that

$$
\begin{aligned}
& Z C_{1}^{*}(T)-Z C_{1}^{*}\left(T^{\prime}\right)=s t_{T_{H}}(z)\left(d_{S_{p, q+1}}(z)-d_{S_{1, p+q}}(a)\right)+ \\
& +d_{T_{H}}(z)\left(s t_{S_{p, q+1}}(z)-s t_{S_{1, p+q}}(a)\right) .
\end{aligned}
$$

We have $d_{S_{p, q+1}}(z)=d_{S_{1, p+q}}(a)=1, s_{S_{p, q+1}}(z)=q+$ $2+q+1=2 q+3$ and $s t_{S_{1, p+q}}(a)=p+q+p+q+1=$ $2 p+2 q+1>2 q+3$.

It follows that $Z C_{1}^{*}\left(T^{\prime}\right)>Z C_{1}^{*}(T)$, hence in this case again $T$ does not maximize the $Z C_{1}^{*}$-index.

By the previous cases, the trees with $k$ pendants that maximize the $Z C_{1}^{*}$-index must be spider trees or double stars. Let $T$ be such a tree.

Assume there are in $T$ one pendant path $P$ of length 1 and one pendant path $Q$ of length at least 3 , both rooted in the same vertex $u$. Denote by $x$, respectively $y$, the pendant extremities of the two paths $P$ and $Q$. Denote by $z$ the neighbor of $y$ in $Q$. Then $s_{T}(z)=3$ and $s_{T}(x)=d_{T}(u)=k$. By Corollary 3 and Remark 1 we have $Z C_{1}^{*}(t(T,[y, z], z, x))-Z C_{1}^{*}(T)=2\left(s_{T}(x)-s_{T}(z)\right)+2>$ 0 , thus $Z C_{1}^{*}(T)$ is not maximum in this case.

Since a double star has $k=n-2$ pendants and a spider tree in $\mathcal{T}_{n, k, \leq 2}$ has at most $2 k+1$ vertices, it follows that a tree with $k$ pendants that maximize the $Z C_{1}^{*}$-index is in $\mathcal{T}_{n, k, \leq 2} \cup \mathcal{S}_{n}^{d}$ if $k=n-2$, in $\mathcal{T}_{n, k, \leq 2}$ if $n-1 \leq 2 k, k \neq n-2$ and in $\mathcal{T}_{n, k, \geq 2}$ otherwise (note that the star $S_{n}$ is a spider tree from $\left.\mathcal{T}_{n, n-1, \leq 2}\right)$. If $k=n-2$, we have, by Lemma 4:

$Z C_{1}^{*}(T)=2 n(n-2)-(n-2)^{2}-3(n-2)=(n-2)(n-1)$. By Lemma 3 it follows that $Z C_{1}^{*}(T)=Z C_{1}^{*}(S)$ for any $S \in \mathcal{S}_{n}^{d}$ and $T \in \mathcal{T}_{n, k, \leq 2}$, hence the trees with $k$ pendants in these families maximize the $Z C_{1}^{*}$-index.

Assume now $n-1>2 k$. By Lemma 4 , the result follows.

\section{UNICYCLIC GRAPHS}

\section{A. Unicyclic graphs with fixed girth}

A unicyclic graph is a connected graph containing exactly one cycle. Thus, a unicyclic graph with $n$ vertices has $n$ edges.

Let $n \geq 4$ and $g<n$. In this section we consider unicyclic graphs with $n$ vertices and girth $g$, that is having the length of the cycle equal to $g$.

Denote by $\mathcal{U}_{n, g}$ the family of unicyclic graphs with $n$ vertices and girth $g$ such that any two non-pendant vertices incident to pendant edges are adjacent vertices on the cycle.

Remark 3: Let $n \geq 3$ and $a$ is a vertex in the cycle graph $C_{n}$. Then $\tau_{C_{n}}(a)=\epsilon_{n} / 2$ and

$$
Z C_{1}^{*}\left(C_{n}\right)=n \cdot d_{C_{n}}(a) \cdot \tau_{C_{n}}(a)=n \epsilon_{n}
$$

Lemma 5: Let $n \geq 3$ and $g<n$. Then for every $G \in \mathcal{U}_{n, g}$ we have $Z C_{1}^{*}(G)=(n-g)^{2}+\left(\alpha_{g}+1\right)(n-g)+\epsilon_{g} g$

Next we study the $Z C_{1}^{*}$-index for graphs obtained from a graph $G$ by replacing a pendant subgraph with another sub- 
graph, in order to find transformations that strictly increase or decrease $Z C_{1}^{*}(G)$.

Lemma 6: Let $G$ be a connected graph and $T$ a tree with $n>2$ vertices. Let $a \in V(G)$ such that $a$ has at least one neighbor that is not pendant and let $b \in V(T)$. Let $G_{1}=(G, a) \odot(T, b)$.

1. Consider $G_{2}=(G, a) \odot\left(P_{n}, v\right)$, where $v$ is a pendant vertex in $P_{n}$. Then $Z C_{1}^{*}\left(G_{1}\right) \geq Z C_{1}^{*}\left(G_{2}\right)$ with equality only if $G_{1} \simeq G_{2}$, that is only if $T \simeq P_{n}$ and $v$ is a pendant vertex in $T$.

2. Consider $G_{3}=(G, a) \odot\left(S_{n-1}, c\right)$, where $c$ is the center of star $S_{n-1}$. Then $Z C_{1}^{*}\left(G_{1}\right) \leq Z C_{1}^{*}\left(G_{3}\right)$ with equality only if $G_{1} \simeq G_{3}$, that is only if $T \simeq S_{n-1}$ and $c$ is the center of $T$.

Proof: By Corollary 1 we have

$$
\begin{aligned}
Z C_{1}^{*}\left(G_{1}\right)= & Z C_{1}^{*}(G)+Z C_{1}^{*}(T)+2 s_{T}(b) d_{G}(a)+ \\
& +d_{T}(b)\left(s_{G}(a)+\tau_{G}(a)-d_{G}(a)\right) . \\
Z C_{1}^{*}\left(G_{2}\right)= & Z C_{1}^{*}(G)+Z C_{1}^{*}\left(P_{n}\right)+2 s_{P_{n}}(v) d_{G}(a)+ \\
& +d_{P_{n}}(v)\left(s_{G}(a)+\tau_{G}(a)-d_{G}(a)\right) . \\
Z C_{1}^{*}\left(G_{3}\right)= & Z C_{1}^{*}(G)+Z C_{1}^{*}\left(S_{n-1}\right)+2 s_{S_{n-1}}(c) d_{G}(a)+ \\
& +d_{S_{n-1}}(c)\left(s_{G}(a)+\tau_{G}(a)-d_{G}(a)\right) .
\end{aligned}
$$

Since $a$ has at least one neighbor of degree at least 2, then $s_{G}(a)>d_{G}(a)$ and thus $s_{G}(a)+\tau_{G}(a)-d_{G}(a)>0$. In order to compare $Z C_{1}^{*}\left(G_{1}\right)$ with $Z C_{1}^{*}\left(G_{i}\right), i=2,3$ it suffices to compare $Z C_{1}^{*}(T)$ with $Z C_{1}^{*}\left(P_{n}\right)$, respectively $Z C_{1}^{*}\left(S_{n-1}\right)$, and coefficients of $s_{G}(a)+\tau_{G}(a)-d_{G}(a)$ and $d_{G}(a)$.

1. First note that, since $n>2$, every vertex $x \in V(T)$ has $s_{T}(x) \geq 2$ since it either has at least two neighbors or is pendant, and in this case its neighbor has degree at least 2. Since $n>2$, we have

- $d_{T}(b) \geq 1=d_{P_{n}}(v), s_{T}(b) \geq 2=s_{P_{n}}(v)$;

- $Z C_{1}^{*}(T) \geq Z C_{1}^{*}\left(P_{n}\right)$ with equality only if $T \simeq P_{n}$ (Theorem 2).

If $T \not P_{n}$ then $Z C_{1}^{*}(T)>Z C_{1}^{*}\left(P_{n}\right)$, hence $Z C_{1}^{*}\left(G_{1}\right)>$ $Z C_{1}^{*}\left(G_{2}\right)$. Otherwise, $T$ is a path. If $b$ is not an extremity of $T$, then $d_{T}(b)>1=d_{P_{n}}(v)$ and again we obtain $Z C_{1}^{*}\left(G_{1}\right)>Z C_{1}^{*}\left(G_{2}\right)$.

2. We have:

- $d_{T}(b) \leq n-1=d_{S_{n-1}}(c)$

- $s_{T}(b)=\tau_{T}(b)+d_{T}(b) \leq n-1=s_{S_{n-1}}(c)$

- $Z C_{1}^{*}(T) \leq Z C_{1}^{*}\left(S_{n-1}\right)$ with equality only if $T \in \mathcal{S}_{n}$ (Theorem 1).

If $d_{T}(b)<n-1$ then $Z C_{1}^{*}\left(G_{1}\right)<Z C_{1}^{*}\left(G_{3}\right)$.

Otherwise $T \simeq S_{n-1}$ and $b$ is the center of $T$.

Theorem 4: Let $n \geq 5$ and $g<n$. The unicyclic graphs with $n$ vertices and girth $g$ having the maximum value of modified first Zagreb connection index are the graphs in $\mathcal{U}_{n, g}$.

Proof: Let $G$ be a unicyclic graph with $n$ vertices and girth $g$ such that $G \notin \mathcal{U}_{n, g}$.

Case 1. There are in $G$ two non-pendant vertices $u, y$ both incident to at least one pendant edge, such that $d(u, y) \geq 2$.
Assume wlog that $s_{G}(u)+d_{G}(u)+\tau_{G}(u) \geq s_{G}(y)+d_{G}(y)+$ $\tau_{G}(y)$ and let $y x$ be a pendant edge incident in $y$. Consider the graph $G^{\prime}=t(G, P, y, u)$, where $P=[y, x]$. Note that $G^{\prime}$ is also an unicyclic graph of girth $g$. By Corollary 3 we have $Z C_{1}^{*}\left(G^{\prime}\right)-Z C_{1}^{*}(G)=\left(s_{G}(u)+d_{G}(u)+\tau_{G}(u)\right)-$ $\left.\left(s_{G}(y)+d_{G}(y)+\tau_{G}(y)\right)\right)+2>0$, hence $G$ is not maximal with respect to $Z C_{1}^{*}$ in this case.

Consider now that $G$ is not in case 1 , that is either the pendant edges are incident in the same vertex or any two vertices in which are incident pendant edges are adjacent.

Case 2. All the vertices of the cycle of $G$ have degree 2 , with one exception. Then $G$ is a vertex-union of a tree $T_{G}$ with $n-g+1$ vertices and a cycle of length $g: G=$ $\left(C_{g}, y\right) \odot\left(T_{G}, y\right)$. Consider $H=\left(C_{g}, y\right) \odot\left(S_{n-g}, a\right)$ where $a$ is the center of star $S_{n-g}$. Then $H \in \mathcal{U}_{n, g}$, hence $G \nsucceq H$. By Lemma 6 we obtain $Z C_{1}^{*}(G)<Z C_{1}^{*}(H)$.

Case 3. Assume $G$ is not in any of cases 1 and 2. Then any two vertices in which are incident pendant edges are adjacent and belong to the cycle of $G$, hence $G \in \mathcal{U}_{n, g}$.

It follows that the unicyclic graphs that maximize $Z C_{1}^{*}$ belong to the family $\mathcal{S}_{n}$. By Lemma 3 all these graphs have the same value of $Z C_{1}^{*}$, hence they maximize the first modified Zagreb connection index.

Let $G$ be a unicyclic graph and $C$ its unique cycle. Note that the connected components of $G-E(C)$ are trees rooted in vertices belonging to $C$. Also, if the components that have at least 2 vertices are pendant subgraphs of $G$ with the root on cycle $C$.

In order to obtain unicyclic graphs with smaller $Z C_{1}^{*}$ index we study the changes of this index when replacing such a component with paths (with the same number of vertices) rooted in one extremity, using Lemma 6. Moreover, if one such component is a path, consider the graph obtained by attaching this component to a pendant vertex of $G$ instead of a vertex from cycle $C$. The following result holds.

Lemma 7: Let $G$ be a connected graph containing a cycle $C$ such that $G-E(C)$ is a forest with all components paths having one extremity in $V(C)$ and at least one component is not trivial (that is $G$ is not cycle graph). Let $a$ be a vertex of $G$ belonging to cycle $C$ and $b$ a pendant vertex in $G$. Consider graphs $G_{1}=(G, a) \odot\left(P_{n}, v\right)$, $G_{2}=(G, b) \odot\left(P_{n}, v\right)$, where $v$ is a pendant vertex in $P_{n}$. Then $Z C_{1}^{*}\left(G_{1}\right)>Z C_{1}^{*}\left(G_{2}\right)$.

Proof: We compare $Z C_{1}^{*}\left(G_{1}\right)$ and $Z C_{1}^{*}\left(G_{2}\right)$ using the formulas given by Lemma 1:

$$
\begin{aligned}
Z C_{1}^{*}\left(G_{1}\right)= & Z C_{1}^{*}(G)+Z C_{1}^{*}\left(P_{n}\right)+ \\
& +d_{P_{n}}(v)\left(s_{G}(a)+\tau_{G}(a)\right)+ \\
& +\left(s_{P_{n}}(v)+\tau_{P_{n}}(v)\right) d_{G}(a) \\
Z C_{1}^{*}\left(G_{2}\right)= & Z C_{1}^{*}(G)+Z C_{1}^{*}\left(P_{n}\right)+ \\
& +d_{P_{n}}(v)\left(s_{G}(b)+\tau_{G}(b)\right)+ \\
& +\left(s_{P_{n}}(v)+\tau_{P_{n}}(v)\right) d_{G}(b)
\end{aligned}
$$

Note that $s_{P_{n}}(v)+\tau_{P_{n}}(v)>0$ and $d_{P_{n}}(v)>0$, hence it suffices to compare $s_{G}(a)+\tau_{G}(a)$ with $s_{G}(b)+\tau_{G}(b)$ and 
$d_{G}(a)$ with $d_{G}(b)$.

Since $a \in C$, we have $d_{G}(a) \geq 2>d_{G}(b), s_{G}(a) \geq 4$.

Let $u$ be the unique neighbor of $b$.

If $u \notin V(C)$, then $u$ is a vertex of degree 2 and $s_{G}(b)+$ $\tau_{G}(b)=d_{G}(u)+1=3$. Otherwise $u$ has degree 3 in $G$ and the vertices at distance 2 from $b$ are the two neighbors of $u$ in $C$, hence in this case $s_{G}(b)+\tau_{G}(b)=3+2=5$. In both cases we have $s_{G}(b)+\tau_{G}(b) \leq 5$.

If $s_{G}(a)+\tau_{G}(a) \geq 5 \geq s_{G}(b)+\tau_{G}(b)$, hence, since $d_{G}(a)>d_{G}(b)$, we obtain $Z C_{1}^{*}\left(G_{1}\right)>Z C_{1}^{*}\left(G_{2}\right)$.

It remains to consider the case when $s_{G}(a)+\tau_{G}(a) \leq 4$. But $s_{G}(a) \geq 4$, which implies $\tau_{G}(a)=0$. Since $a \in V(C)$, this is possible only if $C$ is a cycle of length 3 . But in this case, since $G$ is not a cycle graph, then either $a=u$ or $d_{G}(a, b)>1$.

If $d_{G}(a, b)>1$, then $\tau_{G}(a) \geq 1$, contradiction.

If $a=u$ then $d_{G}(a)=3$ and $s_{G}(a)=5$ ( $a$ has two neighbors on $C$ and is also adjacent to $b$ ), hence again we have $s_{G}(a)+\tau_{G}(a) \geq 5$, contradiction.

Hence the case $s_{G}(a)+\tau_{G}(a) \leq 4$ cannot occur.

Theorem 5: Let $n \geq 5$ and $g<n$. The unicyclic graph with $n$ vertices and girth $g$ having the minimum value of modified first Zagreb connection index is $T_{g, n-g}=$ $\left(C_{g}, a\right) \odot\left(P_{n-g+1}, v\right)$, where $v$ is a pendant vertex in $P_{n-g+1}$ (the $(g, n-g)$-tadpole graph). Moreover,

$$
Z C_{1}^{*}\left(T_{g, n-g}\right)= \begin{cases}4(n-g)+\epsilon_{g} g+\alpha_{g}, & \text { if } g \leq n-2 \\ \epsilon_{g} g+\alpha_{g}+2, & \text { if } g=n-1\end{cases}
$$

Proof: Let $G$ be a unicyclic graph with $n$ vertices and girth $g$. Let $C$ be the unique cycle of $G$. Assume that $G \not$ $\left(C_{g}, a\right) \odot\left(P_{n-g+1}, v\right)$. Then $G$ is in one of the following cases.

Case 1. At least one of the connected components in $G-$ $E(C)$ is not a path with one extremity in $V(C)$.

Denote by $T$ one of these components (which is a tree) and by $p$ its number of vertices. Let $v$ be the unique vertex in $V(T) \cap V(C)$ and $G_{e}=G-(T-v)$. Then $G=\left(G_{e}, v\right) \odot$ $(T, v)$. Consider $G_{1}=\left(G_{e}, v\right) \odot\left(P_{p}, u\right)$, where $u$ is pendant in $P_{p}$. Note that $G_{1}$ is also a unicyle graph of girth $g$ and $v$ has at least one non-pendant neighbor. Then, by Lemma 6, $Z C_{1}^{*}\left(G_{1}\right)<Z C_{1}^{*}(G)$, hence $G$ is not minimal regarding to $Z C_{1}^{*}$.

Case 2. All of the connected components in $G-E(C)$ are paths with one extremity in $V(C)$ and at least two of the components have more than one vertex. Let $T_{1}$, $T_{2}$ be two such components. Let $v_{1}$ be the unique vertex in $V\left(T_{1}\right) \cap V(C)$ and $G_{e}=G-\left(T_{1}-v\right)$. Then $G=\left(G_{e}, v_{1}\right) \odot\left(T_{1}, v_{1}\right)$. Let $v_{2}$ be the extremity of $T_{2}$ that is not in $V(C)$. Consider $G_{2}=\left(G_{e}, v_{2}\right) \odot\left(T_{1}, v_{1}\right)$. By Lemma $7 Z C_{1}^{*}\left(G_{1}\right)<Z C_{1}^{*}(G)$, hence again $G$ is not minimal regarding to $Z C_{1}^{*}$.

It follows that $G$ does not minimize $Z C_{1}^{*}$ if it is in Case 1 or Case 2 . Hence $T_{g, n-g}=\left(C_{g}, a\right) \odot\left(P_{n-g+1}, v\right)$ is the only unicyclic graph with $n$ vertices and girth $g$ having minimum value of $Z C_{1}^{*}$. By Corollary 2 the value of $Z C_{1}^{*}\left(T_{g, n-g}\right)$ is

$$
\begin{cases}Z C_{1}^{*}\left(P_{n-g+1}\right)+\epsilon_{g} g+\alpha_{g}+2 \cdot 3, & \text { if } n-g+1 \geq 3 \\ 0+\epsilon_{g} g+\alpha_{g}+2 \cdot 1, & \text { if } g=n-1 .\end{cases}
$$

The result follows by Theorem 2 , since $Z C_{1}^{*}\left(P_{n-g+1}\right)=$ $4(n-g+1)-10$.

\section{B. Unicyclic graphs with arbitrary girth}

In previous section extremal unicyclic graph with fixed girth were determined. We use the results on extremal unicyclic graph with fixed girth to study how the maximum and minimum value of $Z C_{1}^{*}$ evolves when girth is variable, in particular to find the extremal unicyclic graphs with $n$ vertices.

Theorem 6: Let $n \geq 5$. The unicyclic graphs with $n$ vertices having the maximum value of modified first Zagreb connection index are the graphs in $\mathcal{U}_{n, 5}$ if $n<8$ (having $Z C_{1}^{*}(G)=n^{2}-3 n+10$ ), in $\mathcal{U}_{n, 5} \cup \mathcal{U}_{n, 3}$ if $n=8$ (having $Z C_{1}^{*}(G)=n^{2}-3 n+10$ ) and in $\mathcal{U}_{n, 3}$ if $n>8$ (having $\left.Z C_{1}^{*}(G)=n^{2}-n-6\right)$.

Proof: Let $n \geq 5$. By Theorem 4 and Lemma 5, it suffices to find the girth $g$ for which the formula from Lemma 5 is maximum (for a fixed $n$ ).

For $g \geq 5$, the value $(n-g)^{2}+7(n-g)+4 g$ is maximum for $g=5$ and is equal to $n^{2}-3 n+10$.

For $g=4$ the maximum $Z C_{1}^{*}$ is $(n-g)^{2}+6(n-g)+2 g=$ $n^{2}-2 n$, and for $g=3$ is $(n-g)^{2}+5(n-g)=n^{2}-n-6$.

By comparing the maximum values of for $g=3,4,5$ the result follows.

Theorem 7: Let $n \geq 5$. The unicyclic graph with $n$ vertices having the minimum value of modified first Zagreb connection index is $T_{3, n-3}$.

Proof: The result follows from Theorem 5.

\section{CONNECTED GRAPHS WITH $n$ VERTICES}

One important problem is to find the structure of an extremal connected graph with respect to $Z C_{1}^{*}$, having $n$ vertices and no other constrains, that is to know the possible structure of molecules with $n$ atoms that have minimum or maximum value of the $Z C_{1}^{*}$-index.

The following results on existence of regular and almost regular graphs with given degree sequence are well known.

Lemma 8: 1) Let $p, n$ be two positive integers such that $p \leq n-1$. Then there is a graph with degree sequence $\left\{p^{n}\right\}$ if and only if the product $p n$ is even.

2) Let $p, n_{1}, n_{2}$ be positive integers such that $p \leq n_{1}+$ $n_{2}-2$. Then there is a graph with degree sequence $\left\{p^{n_{1}},(p+1)^{n_{2}}\right\}$ if and only if $p n_{1}+(p+1) n_{2}$ is even.

Lemma 9: Let $G$ be a graph with $n$ vertices. If any vertex $v$ has degree at least $\left[\frac{n}{2}\right]$, then $\operatorname{diam}(G) \leq 2$.

Proof: First note that $G$ is connected, since each connected component must have $\left[\frac{n}{2}\right]+1$ vertices.

Assume by contradiction that there is a vertex $v$ with $e c c(v)>2$. Let $u$ be a vertex such that $d(v, u)=e c c(v)$ 
and $P$ be a path of length $d(u, v)$ (geodesic) between $u$ and $v$. Then $u$ and $v$ have no common neighbors and each is adjacent with exactly one vertex from $P$. It follows that we must have

$|V(G)-V(P)| \geq d_{G}(v)-1+d_{G}(u)-1 \geq 2\left[\frac{n}{2}\right]-2 \geq n-3$.

But

$|V(G)-V(P)|=n-(d(v, u)+1)=n-e c c(v)-1 \leq n-4$, contradiction.

Theorem 8: Let $n \geq 5$.

1) The complete graph $K_{n}$ is the unique graph that minimizes the index $Z C_{1}^{*}$ among all $n$-vertex connected graphs.

2) A connected graph $G$ with $n$ vertices maximizes the index $Z C_{1}^{*}$ among all $n$-vertex connected graphs if and only if it has diameter 2 and: noitemsep,nolistsep

- $d(G) \subseteq\left\{\frac{n}{2}-1, \frac{n}{2}\right\}$, if $n \in\{4 k, 4 k+2 \mid k \in \mathbb{N}\}$

- $d(G)=\left\{\frac{n-1}{2}\right\}$, if $n \in\{4 k+1 \mid k \in \mathbb{N}\}$

- there exists a vertex $x \in V(G)$ such that $d(G) \backslash$ $\{d(x)\}=\left\{\frac{n-1}{2}\right\}$ and $d(x) \in\left\{\frac{n-1}{2}-1, \frac{n-1}{2}+1\right\}$, if $n \in\{4 k+3 \mid k \in \mathbb{N}\}$

Proof:

1) We have $Z C_{1}^{*}\left(K_{n}\right)=0$. Moreover, a graph $G$ has $Z C_{1}^{*}(G)=0$ if and only if it has diameter 1 , that is if and only if is complete.

2) Let $G$ be a $n$-vertex connected graph. For a vertex $v$ of $G$ we have $d_{G}(v) \tau_{G}(v) \leq d_{G}(v)\left(n-1-d_{G}(v)\right)$ with equality only if $\operatorname{ecc}_{G}(v)=2$. It follows that

$$
Z C_{1}^{*}(G) \leq \sum_{v \in V(G)} d_{G}(v)\left(n-1-d_{G}(v)\right)
$$

with equality only if $\operatorname{diam}(G)=2$.

Consider three cases, according to parity of $n$.

Case 1. Assume $n$ is even. Then $d_{G}(v)(n-1-$ $\left.d_{G}(v)\right) \leq \frac{n(n-2)}{4}$ with equality only if $\operatorname{ecc}_{G}(v)=2$ and $d_{G}(v) \in\left\{\frac{n}{2}, \frac{n}{2}-1\right\}$ for every $v \in V(G)$. It follows that $Z C_{1}^{*}(G) \leq n \frac{n(n-2)}{4}$ with equality only if $\operatorname{diam}(G)=2$ and every vertex of $G$ has degree $\frac{n}{2}$ or $\frac{n}{2}-1$. Then, if it is not empty, the family of graphs with these properties maximize $Z C_{1}^{*}(G)$. Since $n \cdot \frac{n}{2}$ is even, by Lemma 8 there are $\frac{n}{2}$-regular graphs with $n$ vertices and, by Lemma 9, they have diameter 2 .

Case 2. Assume $n=4 k+1$. Then $d_{G}(v)(n-1-$ $\left.d_{G}(v)\right) \leq \frac{(n-1)^{2}}{4}$ with equality only if $\operatorname{ecc}_{G}(v)=2$ and $d_{G}(v)=\frac{n-1}{2}$ for every $v \in V(G)$. It follows that $Z C_{1}^{*}(G) \leq n \frac{(n-1)^{2}}{4}$ with equality only if $\operatorname{diam}(G)=2$ and every vertex of $G$ has degree $\frac{n-1}{2}$. By Lemma 9 all graphs $G$ with $n$ vertices and $d(G)=\left\{\frac{n-1}{2}\right\}$ have diameter 2 . Since $n \frac{n-1}{2}$ is even, by Lemma 8 , such regular graphs exist.

Case 3. Assume $n=4 k+3$ is even. As in previ- ous case, $Z C_{1}^{*}(G) \leq n \frac{(n-1)^{2}}{4}$ with equality only if $\operatorname{diam}(G)=2$ and every vertex has degree $\frac{n-1}{2}=$ $2 k+1$. But such graph does not exists, since the sum of degrees would be $n(2 k+1)$, which is an odd number. If follows that in this case $Z C_{1}^{*}(G) \leq n \frac{(n-1)^{2}}{4}-1$ with equality only if every vertex has eccentricity 2 and degree $\frac{n-1}{2}=2 k+1$ with one exception $v$, for which $d_{G}(v) \tau_{G}(v)=\frac{(n-1)^{2}}{4}-1$; this is possible only if degree of $v$ is $2 k$ or $2 k+2$ and $d_{G}(v)+\tau_{G}(v)=$ $n-1$, that is $\operatorname{ecc}(v)=2$. Then $Z C_{1}^{*}(G)=n \frac{(n-1)^{2}}{4}-1$ only if $\operatorname{diam}(G)=2$ and there exists a vertex $x \in V(G)$ such that $d(G) \backslash\{d(x)\}=\left\{\frac{n-1}{2}\right\}$ and $d(x) \in\left\{\frac{n-1}{2}-1, \frac{n-1}{2}+1\right\}$. The family of graphs with such properties is not empty, since, by Lemma 8 , there exist graphs with $n$ vertices and degree sequence $\left\{\left(\frac{n-1}{2}\right)^{n-1}, \frac{n-1}{2}+1\right\}$ (the sum of degrees is even) and, by Lemma 9, such graphs have diameter 2 .

\section{CONCLUSIONS}

A common idea to study extremal graphs with respect to a certain index is determining transformations which, applied on a graph, strictly decrease or increase the index. In this paper we proposed a method that allows to find more easily such transformations, based on vertex-union operation. This is useful in obtaining extremal graphs for various families of graphs studied in chemistry. Further studies based on this method can be done on other graphs, such as cacti graphs.

\section{REFERENCES}

[1] A. Ali and N. Trinajstić. A novel/old modification of the first Zagreb index. https://arxiv.org/pdf/1705.10430.pdf.

[2] A. T. Balaban and O. Ivanciuc. Historical Development of Topological Indices. Topological Indices and Related Descriptors in QSAR and QSPR, J. Devillers and A. T. Balaban (Eds.), Gordon and Breach Science Publisher, Amsterdam: 21-57, 1999.

[3] B. Bollobás and P. Erdös. Graphs of extremal weights. Ars Comb., 50: 225-233, 1998.

[4] I. Garcia, Y. Vall and G. Gomez. Using Topological Indices to Predict Anti-Alzheimer and Anti-Parasitic GSK3 Inhibitors by Multi-Target QSAR in Silico Screening. Molecules, 15: 5408-5422, 2010.

[5] H. Gonzales-Diaz and C.R. Munteanu. Topological indices for Medical Chemistry, Biology, Parasitology, Neurological and Social Networks. Transworld Research Network, Kerala, 2010.

[6] I. Gutman and N. Trinastić. Graph theory and molecular orbitals. Total $\pi$ - electron energy of alternant hydrocarbons. Chem Phys. Lett., 17: 535-538, 1972.

[7] S. Nikolić, G. Kovačević, A. Miličević and N. Trinajstić The Zagreb Indices 30 Years After. Croat. Chem. Acta, 76: 113-124, 2003.

[8] R. Todeschini and V. Consoni New local vertex invariants and molecular descriptors based on functions of the vertex degrees. MATCH, 64: 359-372, 2010.

[9] B. Zhou and N. Trinajstic Some properties of the reformulated Zagreb indices. J. Math. Chem., 48(3): 714-719, 2010. 